\title{
Preliminary study of $B_{B}$ parameter using Lattice NRQCD
}

\author{
N. Yamada ${ }^{\mathrm{a} *}$, S. Hashimoto ${ }^{\mathrm{b}, \mathrm{d}}$, K-I. Ishikawa ${ }^{\mathrm{a}}$, H. Matsufuru ${ }^{\mathrm{c} \dagger}$, T. Onogi ${ }^{\mathrm{a}}$ and S. Tominaga $^{\mathrm{d} \dagger}$ \\ ${ }^{a}$ Department of Physics, Hiroshima University, Higashi-Hiroshima 739-8526, Japan \\ ${ }^{\mathrm{b}}$ Fermi National Accelerator Laboratory, P.O. Box 500, Batavia, IL 60510 \\ cInstitut für Theoretische Physik, Universität Heidelberg, Heidelberg, Germany \\ ${ }^{\mathrm{d}}$ High Energy Accelerator Research Organization(KEK),Tsukuba 305-0801, Japan
}

We present the preliminary result of the calculation of $B_{B}$ parameter using lattice NRQCD. The calculation is performed on a $16^{3} \times 48$ quenched lattice at $\beta=5.9$ with clover light quark. The use of lattice NRQCD enables us to investigate the $1 / m_{Q}$ correction from the static limit. The observed mass dependence is well described by the vacuum saturation.

\section{Introduction}

The theoretical determination of the $B$ meson decay constant $f_{B}$ and the bag parameter $B_{B}$ are necessary to constrain $\left|V_{t b}^{*} V_{t d}\right|$. The lattice technique has been considered to be the most reliable approach, and a number of authors have calculated these quantities in different approaches. In recent years, the value of $f_{B}$ is almost being settled (but still within the quenched approximation) [1]. On the other hand, $B_{B}$ has been calculated only under the approximation of $m_{b} \rightarrow \infty$ or $a^{-1} \gg m_{b}$, and there has been no systematic study of its heavy quark mass dependence. In this talk we study the $1 / m_{Q}$ correction to the static limit using the NRQCD action, which is the first step to obtain $B_{B}$ with the realistic $b$-quark mass.

\section{Method}

Since the chiral symmetry is broken on the lattice, several lattice operators contribute to $B_{B}$ through

$B_{B}\left(m_{b}\right)=\sum_{i} Z_{i} B_{i}^{\text {lat }}$

\footnotetext{
*Presented by N.Yamada.

${ }^{\dagger}$ H.M. and S.T. would like to thank the JSPS for Young Scientists for a research fellowship.

${ }^{\ddagger}$ T.O. is supported by the Grants-in-Aid of the Ministry of Education (No. 10740125).
}

where $B_{i}^{\text {lat }}$ are

$$
B_{i}^{\text {lat }}\left(a^{-1}\right)=\frac{\left\langle\overline{B^{0}}\left|\hat{\mathcal{O}}_{i}^{l}\left(a^{-1}\right)\right| B^{0}\right\rangle}{\frac{8}{3}\left(\frac{f_{B} M_{B}}{Z_{A}\left(q^{*}\right)}\right)^{2}},
$$

and operators are given by

$$
\begin{aligned}
\hat{\mathcal{O}}^{l}{ }_{L}= & \bar{b} \gamma_{\mu}\left(1-\gamma_{5}\right) d \bar{b} \gamma_{\mu}\left(1-\gamma_{5}\right) d \\
\hat{\mathcal{O}}_{R}{ }_{R}= & \bar{b} \gamma_{\mu}\left(1+\gamma_{5}\right) d \bar{b} \gamma_{\mu}\left(1+\gamma_{5}\right) d \\
\hat{\mathcal{O}}^{l}{ }_{N}= & 2 \bar{b} \gamma_{\mu}\left(1-\gamma_{5}\right) d \bar{b} \gamma_{\mu}\left(1+\gamma_{5}\right) d \\
& +4 \bar{b}\left(1-\gamma_{5}\right) d \bar{b}\left(1+\gamma_{5}\right) d \\
\hat{\mathcal{O}}^{l}{ }_{S}= & \bar{b}\left(1-\gamma_{5}\right) d \bar{b}\left(1-\gamma_{5}\right) d .
\end{aligned}
$$

The operators other than $\hat{\mathcal{O}}^{l} L$ appear with $O\left(\alpha_{s}\right)$ mixing coefficients. Since the one-loop calculation of the coefficients $Z_{i}$ has not yet been done for the NRQCD action, we use those in the static approximation instead in this preliminary study. $Z_{i}^{\prime} s$ at $\beta=5.9$ are given in Table 1 .

The method of the calculation of $B_{i}^{\text {lat's }}$ is standard. We place a point source of the heavy and light quarks at the origin, where the 4 -fermi

\begin{tabular}{|c||c|c|c|c|c|}
\hline$q^{*}$ & $Z_{L}^{\text {stat }}$ & $Z_{R}^{\text {stat }}$ & $Z_{N}^{\text {stat }}$ & $Z_{S}^{\text {stat }}$ & $Z_{A}^{\text {stat }}$ \\
\hline \hline$\pi / a$ & 0.938 & -0.007 & -0.080 & -0.139 & 0.864 \\
$1 / a$ & 0.998 & -0.011 & -0.132 & -0.120 & 0.800 \\
\hline
\end{tabular}

Matching factors at $\beta=5.9$. 


\begin{tabular}{|c|l|}
\hline Size & $16^{3} \times 48 \quad(t=[-24,23])$ \\
\hline Gauge & $\begin{array}{l}\text { Wilson plaquette action } \\
\text { quenched } 100 \text { config. } \\
\beta=5.9, \text { fixed to Coulomb gauge }\end{array}$ \\
\hline Light quark & $\begin{array}{l}\text { SW-Clover action }\left(c_{\mathrm{sw}}=1 / u_{0}^{3}\right) \\
\text { local source at } t=0 \\
\text { KLM norm. [5] }\end{array}$ \\
\hline Heavy quark & $\begin{array}{l}\text { Tadpole improved NRQCD } \\
O(1 / m) \text { and } O\left(1 / m^{2}\right)[6] \\
\\
\text { local source at } t=0\end{array}$ \\
\hline
\end{tabular}

Table 2

Actions and simulation parameters

operators are constructed, and $B$ and $\bar{B}$ mesons propagate in the opposite direction to each other. The ground state extraction is rather easier for NRQCD than for the static approximation, even though we use the local sink. This is another advantage of introducing the $1 / m_{Q}$ corrections.

The actions and lattice parameters are summarized in Table 2. The inverse lattice spacing is determined from the rho meson mass as $a^{-1}=1.62$ $\mathrm{GeV}$. For the heavy quark, we performed two sets of calculations with $1 / m_{Q}$ and $1 / m_{Q}^{2}$ NRQCD actions as in Ref.[6], in order to estimate the higher order effects in the $1 / m_{Q}$ expansion.

\section{Results}

In Figure 1 we plot the $1 / M_{P}$ dependence of $B_{i}^{\text {lat }}$ 's, with $M_{P}$ the pseudoscalar heavy-light meson mass. Since $B_{L}^{\text {lat }}$ should agree with $B_{R}^{\text {lat }}$ with infinite statistics, we take an average of $B_{L}^{\text {lat }}$ and $B_{R}^{\text {lat }}$ in our simulation. $B_{N}$ and $B_{S}$ have significant slopes in $1 / M_{P}$, while $B_{L, R}$ are almost flat. The curvature in $1 / M_{P}$, on the other hand, is small in any case, and the difference between the two different NRQCD actions is negligible. An extrapolation to the static limit does not seem to agree with the result of [8] (at $\beta=6.0$ ) for $B_{N}$ and for $B_{S}$, although it is not quite clear whether this is an inconsistency, with our large statistical errors.

This $1 / M_{P}$ dependence can be roughly understood by the factorization hypothesis (or vacuum saturation) as follows. If the factorization is exact on the lattice, $B_{L, R}^{(\mathrm{fac})}$ becomes unity by definition (solid line in Figure 1). For $B_{N, S}^{(\mathrm{fac})}$, after a little
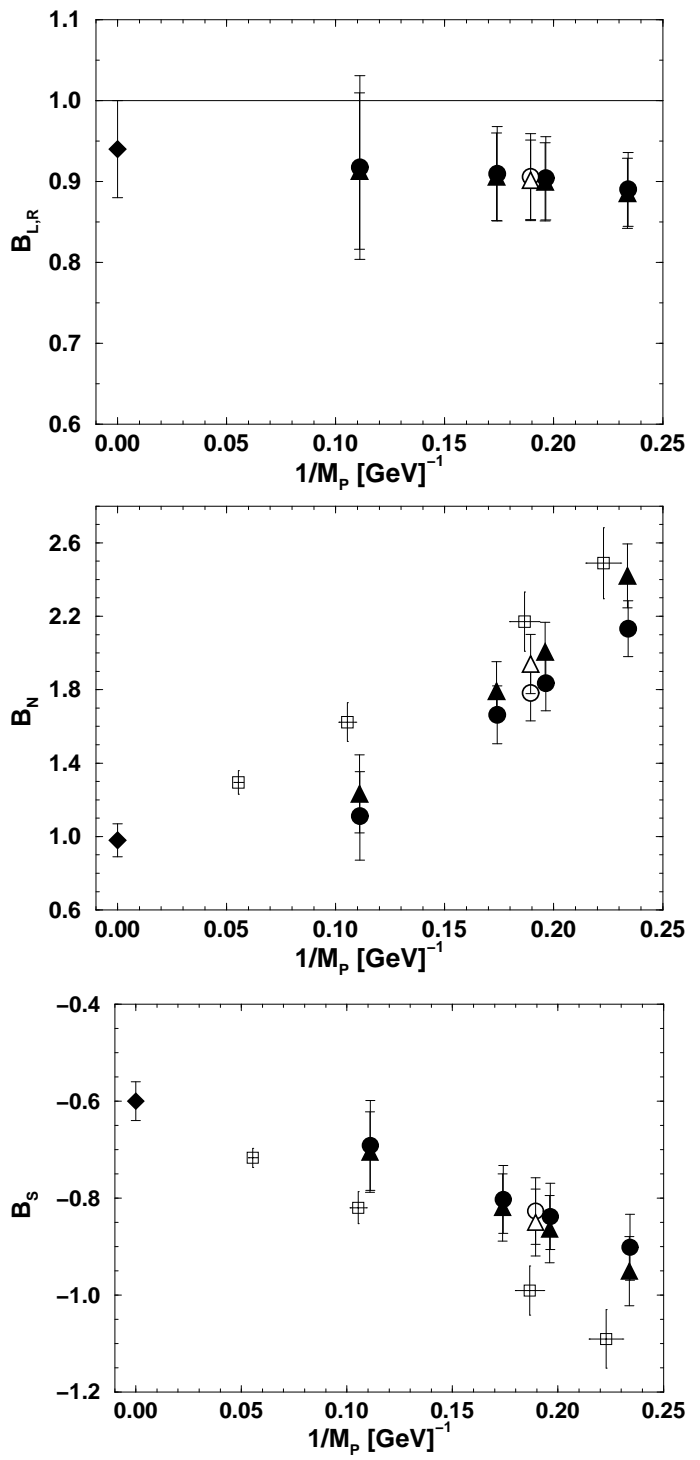

Figure 1. The heavy quark mass dependence of $B_{i}^{\text {lat }}$. The circles are for $O\left(1 / m_{Q}\right)$ calculation and the triangles for $O\left(1 / m_{Q}^{2}\right)$. Opaque symbols are obtained by interpolations to the physical $B$ meson mass, respectively. Diamonds are the results obtained in Ref. [7]. 
algebra, we obtain

$$
\begin{aligned}
B_{N}^{(\mathrm{fac})} & =1-4 \frac{\delta f_{P}^{(1)}}{f_{P}} \\
B_{S}^{(\mathrm{fac})} & =-\frac{5}{8}\left[1-8 \frac{\delta f_{P}^{(1)}}{f_{P}}\right]
\end{aligned}
$$

where $\delta f_{P}^{(1)}$ is the $O\left(1 / m_{Q}\right)$ correction to the decay constant $f_{P} . \quad \delta f_{B}^{(1)}$ is known to be rather large, and as an estimate we use our previous calculation with NRQCD [6]. Substituting the value of $\delta f_{B}^{(1)} / f_{B}$ to Eq.(2) and (3), we obtain open squares in Fig.11 for $B_{N, S}^{(\mathrm{fac})}$. The agreement between the full calculation and the factorization is remarkable. Not only their value itself, but also the slope in $1 / m_{Q}$ seem to be well described with the factorization hypothesis.

The $1 / M_{P}$ dependence of $B_{B}\left(m_{b}\right)$ is shown in Figure 2, which shows a little negative slope. This is because of a large cancellation of $1 / M_{P}$ dependence between those in $B_{N}$ and $B_{S}$. In the leading log approximation, with $\Lambda_{Q C D}^{(5)}=0.2 \mathrm{GeV}$, we obtain the scale invariant $B_{B}$ parameter

$\hat{B}_{B_{d}}=\left(\alpha_{s}\left(m_{b}\right)\right)^{-6 / 23} B_{B}\left(m_{b}\right)=1.17(9)(4)(3)$.

The errors are statistical, higher order in perturbation theory, and the higher order in $1 / m_{Q}$ expansion, respectively. For other quantities, we obtain $B_{B_{s}} / B_{B_{d}}=1.00(2)$ and $f_{B_{s}} / f_{B_{d}}=1.16(3)$. Some additional systematic uncertainties, such as $O\left(a^{2}\right), O\left(\alpha_{s} / m\right), O\left(a \alpha_{s}\right)$ and quenching errors still remain to be estimated.

\section{Summary}

To summarize, we find a large $1 / m_{Q}$ effects in the matrix elements $B_{N}^{l a t}$ and $B_{S}^{l a t}$, although the $O\left(1 / m_{Q}^{2}\right)$ correction seems reasonably small. Their large mass dependence can be roughly understood by the factorization hypothesis (or vacuum saturation). In the present analysis, we combine lattice simulation for finite heavy quark mass with matching coefficients in the static limit, which is still unsatisfactory. For the complete understanding of $1 / m_{Q}$ dependence, matching coefficients with finite heavy quark mass are absolutely necessary.

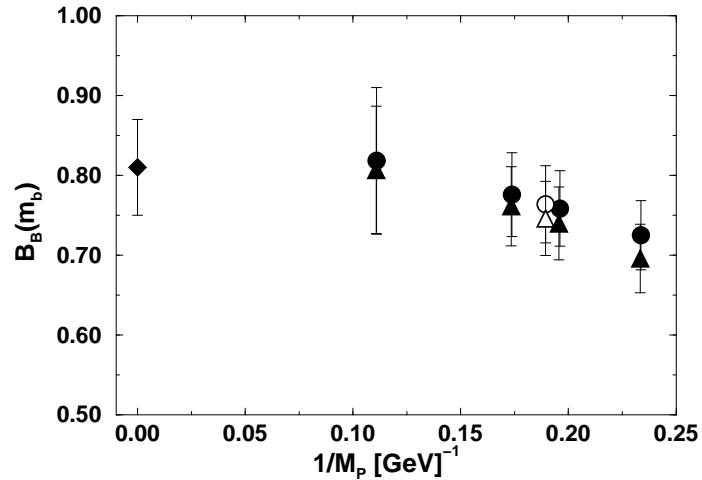

Figure 2. The heavy quark mass dependence of $B_{B}\left(m_{b}\right)$. A diamond is the result of reanalysis 8 . For other symbols, see the previous figure caption.

\section{Acknowledgment}

Numerical calculations have been done on Paragon XP/S at Institute for Numerical Simulations and Applied Mathematics in Hiroshima University. We are grateful to S. Hioki for allowing us to use his program to generate gauge configurations.

\section{REFERENCES}

1. T. Draper, these proceedings.

2. B. A. Thacker and G. P. Lepage, Phys. Rev. D43 (1991) 196; G. P. Lepage et al., Phys. Rev. D46 (1992) 4052.

3. J. Flynn, O. Hernandez and B. Hill, Phys.Rev. D43 (1991) 3709; A. Borrelli and C. Pittori, Nucl.Phys. B385 (1992) 502; M. Ciuchini, E. Franco and V. Gimenez, Phys.Lett. B388 (1996) 167; G Buchalla, Phys.Lett. B395 (1997) 364.

4. J. Christensen, T. Draper and C. McNeile, Phys. Rev. D56 (1997) 6993.

5. G. P. Lepage, Nucl. Phys. B(Proc. Suppl.) 26 (1992) 45; A. X. El-Khadra, A. Kronfeld and P. Mackenzie, Phys. Rev. D55 (1997) 3933.

6. K-I. Ishikawa et al., Phys.Rev. D56 (1997) 7028 .

7. V. Gimenez and G. Martinelli, Phys.Lett. B398 (1997) 135.

8. V. Gimenez and J. Reyes, hep-lat/9806023. 